\title{
Targeting mitochondria in pancreatic cancer
}

Pancreatic ductal adenocarcinoma (PDAC) accounts for the vast majority $(90 \%)$ of all pancreatic cancers, and remains a disease with a very poor prognosis and high morbidity. KRAS mutations are a well-recognized driver event in PDAC, but targeting mutant KRAS is challenging, as patients eventually relapse and existing therapies are not able to eradicate all tumour cells.

Giulio Draetta, Andrea Viale and colleagues have now demonstrated, using a KRAS-inducible mouse model, that a subpopulation of dormant tumour cells survives KRAS-ablation therapy and induce tumour relapse. Importantly, these cells rely on oxidative phosphorylation (OXPHOS) for survival.

"Using this genetic model, we can induce KRAS-driven pancreatic cancer and then, by turning off $K R A S$, induce tumour regression, which is very rapid and takes only a few weeks," explains Viale. The tumours relapsed after 4 to 5 months, indicating that some tumour cells survived the ablation of KRAS signalling. "This mimicked what has been observed in the clinic in patients affected by different tumours receiving new targeted therapies: they almost inevitably succumb to treatment-resistant disease and relapse," continues Viale.

Taking advantage of their mouse model, the researchers, characterized these surviving cells and reported that they are enriched in cancer stem cells and are characterized by strong expression of genes governing mitochondrial function and respiration-thus, relying on OXPHOS for survival. "This was quite surprising, as tumour cells usually rely on aerobic glycolysis to maintain their energetics. Interestingly, surviving cells are not glycolytic at all," says Viale. Of note, the researchers also showed that these surviving cells are specifically eradicated by short exposure to OXPHOS inhibitors, both in vitro and in vivo.

Furthermore, "we demonstrated that cancer cells derived from patients resistant to pharmacological inhibition of the MEK and PI3K signalling pathways, which are engaged by oncogenic KRAS, have

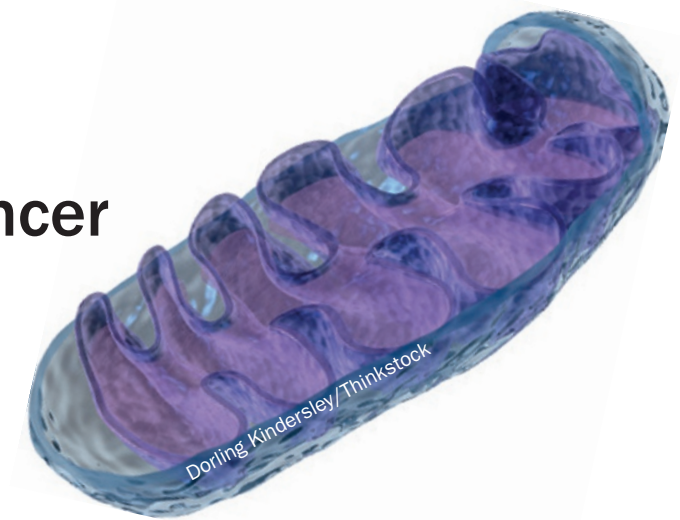

the same metabolic phenotype observed in the surviving cells isolated from the mouse model," says Draetta. "Similarly to the mouse cells, human surviving cells are also extremely sensitive to OXPHOS inhibition," adds Draetta. "These findings are very promising, and we are committed to move this approach towards the clinic," concludes Draetta, as this study provides the rationale for a combined therapeutic strategy targeting both the KRAS pathway and mitochondrial respiration to manage pancreatic cancer.

Alessia Errico

Original article Viale, A. et al. Oncogene ablation-resistan pancreatic cancer cells depend on mitochondrial function. Nature doi:10.1038/nature13611 